\title{
Impacto econômico da obesidade no Brasil
}

\author{
Economic impact of obesity in Brazil
}

\author{
Luciana R. Bahia*, Denizar Vianna Araújo
}

\section{Resumo}

Nas últimas décadas, os gastos em medicina aumentaram de maneira mais significativa do que em outros setores da economia mundial. A incidência crescente do sobrepeso e da obesidade representa um sério problema de saúde pública com implicações para a sociedade e para os sistemas de saúde. As consequências econômicas da obesidade e doenças associadas não se limitam aos elevados custos médicos, mas incluem também os custos indiretos ou sociais, tais como: diminuição da qualidade de vida, problemas de ajustes sociais, perda de produtividade, incapacidade com aposentadorias precoces e morte. Os estudos de custos da doença estimam os custos totais de uma doença para os sistemas de saúde (público e/ou privado), para a sociedade ou para os indivíduos/ familias. Com o conhecimento dos custos relacionados à obesidade, análises econômicas podem ser realizadas para ajudar gestores e formuladores de políticas de saúde a compreender melhor a dimensão do problema e traçar estratégias que melhorem o acesso e o tratamento desses indivíduos, assim como investir em medidas preventivas.

Descritores: Obesidade; Custos e análise de custo; Economia.

\begin{abstract}
Medical expenditures increased more significantly than other sectors of world economy. Obesity is a worldwide epidemic and a serious public health problem with consequences for society and health systems. The economic consequences of obesity are not limited to medical costs, but also include indirect or social costs, such as decreased quality of life, social adjustment problems, productivity loss, premature retirement and death. Cost-ofillness studies try to estimate the total costs of a disease to health systems (public and/or private), to society or individuals/families. With the knowledge of the costs related to obesity, economic analyses can be performed in local reality, helping managers and policy makers to better understand the dimension of the problem and to develop strategies to improve access and treatment of these individuals, as well as how to invest in preventive measures.
\end{abstract}

Keywords: Obesity; Costs and cost analysis; Economics. 


\section{Introdução}

Nas últimas décadas a prevalência da obesidade vem crescendo em quase todo o mundo, sendo considerada uma epidemia global. A Organização Mundial da Saúde (OMS) estima que, em 2005, havia aproximadamente 1,6 bilhões de adultos com sobrepeso e pelo menos 400 milhões de adultos eram obesos em todo o mundo. Há uma previsão de que, em 2015, haverá cerca de 2,3 bilhões de adultos com sobrepeso e mais de 700 milhões com obesidade. ${ }^{1}$ O problema é complexo e exige estratégias preventivas na tentativa de controlar e tratar essa doença e suas complicações.

Os Estados Unidos se destacam pela elevada e crescente prevalência de sobrepeso e obesidade. Segundo um estudo conduzido em 2009 e 2010, a prevalência de obesidade na população adulta foi de cerca de $35 \%$, em ambos os sexos. ${ }^{2}$ A prevalência de sobrepeso e obesidade na população de crianças e adolescentes ficou em torno de $30 \mathrm{e}$ 15\%. Na Inglaterra, dados de 2011 indicam que 1,7\% dos homens e 3,2\% das mulheres já são considerados obesos grau III. ${ }^{3}$ No Brasil, duas pesquisas nacionais de população adulta com dados de peso e estatura autorreferidos (VIGITEL 2006 e 2010) mostraram que as taxas de prevalência de sobrepeso e obesidade têm aumentado, ao longo dos últimos quatro anos, de $43 \%$ para $48,1 \%$, e de $11 \%$ para $15 \%$, respectivamente. ${ }^{4,5}$

Paralelamente, os gastos em medicina aumentaram de maneira mais significativa do que em outros setores da economia mundial. Esse aumento é devido a diversos fatores, tais como: o envelhecimento populacional, com maior necessidade de cuidados de saúde; o surgimento e incorporação de novas tecnologias (medicamentos, testes diagnósticos, procedimentos etc.), e a uma população mais informada com maior demanda por cuidados médicos. Grande parte desses gastos é decorrente das doenças crônico-degenerativas não transmissíveis, as DCNT (diabetes, doenças cardiovasculares, câncer), que são as causas mais comuns de morte no mundo moderno e no Brasil. ${ }^{6}$

As consequências econômicas da obesidade e doenças associadas não se limitam aos elevados custos médicos, mas incluem também os custos indiretos ou sociais, tais como: diminuição da qualidade de vida, problemas de ajustes sociais, perda de produtividade, incapacidade com aposentadorias precoces e morte.

A grande maioria da população brasileira depende exclusivamente do Sistema Único de Saúde (SUS) para obter assistência médica, enquanto apenas $25 \%$ da população geral tem acesso ao Sistema Suplementar de Saúde (aproximadamente 47 milhões de brasileiros). ${ }^{7} \mathrm{O}$ financiamento desses sistemas é complexo e os recursos limitados. $\mathrm{O}$ crescente número de casos de obesidade configura, no longo prazo, uma ameaça à sustentabilidade do tratamento desses indivíduos tanto no âmbito público quanto no privado.

O objetivo dessa revisão é analisar os custos da obesidade e doenças relacionadas no mundo e no Brasil na perspectiva da sociedade e dos sistemas de saúde.

\section{Os custos da obesidade}

O tratamento das doenças relacionadas ao sobrepeso e à obesidade (diabetes, hipertensão arterial, doença coronariana, AVC, osteoartrite e vários tipos de câncer) acarreta um aumento substancial dos gastos de saúde para os diversos sistemas de saúde. Nos Estados Unidos, o gasto per capita com um indivíduo obeso é cerca de 42 a $100 \%$ maior do que um indivíduo com peso normal. ${ }^{8}$ Estima-se que os custos médicos anuais nos EUA chegam a US\$ 147 bilhões para adultos e US\$14,3 milhões para crianças e adolescentes obesos. ${ }^{6}$ A perda de produtividade decorrente do absenteísmo e morte prematura somam aproximadamente US\$ 66 bilhões ao ano. ${ }^{9}$ No Reino Unido, os custos diretos do sobrepeso e obesidade somaram $£ 3,23$ bilhões em 2007 , o que foi equivalente a aproximadamente $5 \%$ dos gastos do Sistema Nacional de Saúde..$^{10}$ Uma revisão sobre 19 estudos econômicos a respeito da obesidade e doenças relacionadas na Europa estimou que os custos diretos incrementais por indivíduo obeso chegam a ser de $€ 117$ a $€ 1.873$ em comparação a um indivíduo de peso normal."

Os gastos com as doenças relacionadas com a obesidade representam uma parte significativa do orçamento de vários países. Na Alemanha, os custos totais da obesidade (custos diretos e indiretos) compreendem 0,47 a $0,61 \%$ do produto interno bruto (PIB). ${ }^{12}$ No Canadá, os custos diretos para o sistema de saúde com as doenças relacionadas ao sobrepeso e à obesidade no ano 
de 2006 foram de \$ 2 bilhões e \$ 3,9 bilhões, representando $1,4 \%$ e $2,6 \%$ dos gastos totais em saúde respectivamente. ${ }^{13}$

\section{Estudos de custos da doença - dados brasileiros}

Os estudos de custos da doença tentam estimar os custos totais de uma doença para os sistemas de saúde (público e/ou privado), para a sociedade ou para os indivíduos/famílias. Distinguem-se basicamente três tipos de custos: custos diretos (médicos e não médicos), custos indiretos (como perda de produtividade) e custos intangíveis (por exemplo a qualidade de vida). Várias combinações de custos são encontradas na literatura e essas são uma escolha dos pesquisadores, dependendo da perspectiva do estudo (pagador, sociedade, hospital). Uma estimativa de custos que incorpore o maior número de componentes de custos será a mais acurada possível.

Para estimar os custos diretos da obesidade, deve-se levantar dados sobre utilização de recursos de saúde nas unidades ambulatoriais e hospitalares. A identificação dos indivíduos com diagnósticos relacionados à obesidade é possível através da codificação internacional de doenças, embora a obesidade não seja um diagnóstico principal muito frequente.

Usualmente não existe uma única fonte de dados através da qual podem ser obtidas todas as informações relevantes sobre utilização de recursos de diagnóstico e tratamento da obesidade e seus custos, havendo necessidade da combinação de diversas fontes de informação. A maioria dos sistemas de saúde possui alguma fonte de dados que pode ser utilizada por pesquisadores e gestores (banco de dados administrativos, internações hospitalares, procedimentos ambulatoriais, registros de pacientes, censos, pesquisas populacionais etc.). No caso de estudos realizados no âmbito do SUS, o site do DATASUS (Banco de dados do Sistema Único de Saúde) oferece informações de valores pagos aos prestadores, porém muitas vezes carece de detalhamento quanto a todos os recursos de saúde utilizados (como medicamentos, materiais médicos etc.), por ser um banco de dados administrativo, não tendo sido criado com fins acadêmicos. O Ministério da
Saúde possui um banco de dados de preços que armazena, sistematiza e disponibiliza todos os preços praticados para medicamentos e produtos em geral (equipamentos, órteses, próteses, testes diagnósticos etc.), denominado Banco de Preços em Saúde (BPS). ${ }^{14}$

Para estimar a contribuição do fator de risco (obesidade) sobre um desfecho de interesse (como diabetes, doença cardiovascular, câncer), frequentemente utiliza-se o cálculo do risco atribuível populacional, ou seja, a proporção dos casos da doença que ocorrem em decorrência da exposição ao fator de risco. Esse cálculo é feito com base nas informações provenientes de estudos observacionais e os riscos relativos de determinados desfechos em indivíduos obesos comparados com não obesos.

No Brasil, três estudos foram conduzidos para demonstrar os custos da obesidade e condições associadas para o SUS através dessa metodologia. ${ }^{15-17}$ Os resultados desses estudos não são comparáveis entre si, pois os métodos utilizados tiveram diferenças, tais como: tipos de custos incluídos (custos diretos, médicos e não médicos, e custos indiretos), estimativas ambulatoriais e/ ou hospitalares, a valoração dos custos e os períodos de análise.

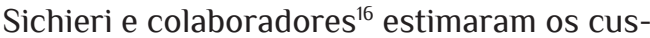
tos de hospitalização relacionados às doenças associadas ao sobrepeso/obesidade através dos dados das hospitalizações de homens e mulheres de 20 a 60 anos do Sistema de Informações Hospitalares do Sistema Único de Saúde (SIH-SUS) para o ano de 2001. Demonstraram que cerca de 3 a 5\% de todas as internações no Brasil foram decorrentes de doenças relacionadas diretamente à obesidade. Além disso, avaliaram também dados de perda de produtividade pela identificação do número de dias de hospitalização. O número de dias de trabalho perdidos em função da obesidade e doenças associadas foi de 3,9 a 10,6 dias/ano. A causa mais comum de perda de produtividade foi o diabetes mellitus, seguido pelas doenças cardiovasculares. ${ }^{9}$

Bahia e colaboradores ${ }^{17}$ demonstraram os custos médicos relacionados ao tratamento ambulatorial e hospitalar de doenças associadas ao sobrepeso e à obesidade nos anos de 2008 a 2010. Com base nos riscos relativos (dados internacionais) ${ }^{18}$ e prevalência de sobrepeso e obesidade 
no Brasil pode-se estimar a fração atribuível da obesidade sobre desfechos de interesse. Estimouse que o SUS gasta anualmente cerca de $R$ \$ 3,6 bilhões por ano com o tratamento dessas doenças, sendo R $\$ 2,4$ bilhões com o tratamento hospitalar (68\%) e R\$ 1,2 bilhões (32\%) com o tratamento ambulatorial (Tabela 1). As doenças cardiovasculares, provavelmente pela maior frequência, foram responsáveis por $67 \%$ dos custos, seguida pelo tratamento do câncer. Considerando que os custos indiretos (perda de produtividade, licenças médicas, morte prematura) não foram incluídos e os dados de gastos médicos foram obtidos através do banco de dados do DATASUS, ou seja, valores reembolsados às unidades de saúde, os autores ressaltam que esses números são uma estimativa bastante conservadora dos gastos públicos com pacientes obesos, já que o custo real do tratamento é classicamente maior do que os valores reembolsados. ${ }^{10}$ Os custos estimados foram equivalentes a $0,09 \%$ do PIB nacional em $2010,{ }^{19}$ muito abaixo dos demonstrados em estudos internacionais.

\section{Conclusão}

Os custos totais decorrentes da obesidade e doenças relacionadas são significativos e crescentes para os sistemas de saúde e para a sociedade. Os dados de custos nacionais são importantes informações que ajudam na tomada de decisão por gestores sobre investimentos e prioridades, auxiliando no processo de eficiência nos gastos em saúde. A análise do impacto orçamentário de intervenções preventivas e terapêuticas permitirá o acesso e a sustentabilidade, em longo prazo, do tratamento desses indivíduos.

\section{Referências}

1. Obesity: preventing and managing the global epidemic. World Health Organ Tech Rep Ser. 2000;894:i-xii, 1-253.

2. Flegal KM, Carroll MD, Kit BK, Ogden CL. Prevalence of obesity and trends in the distribution of Body Mass Index among US adults, 1999-2010. JAMA. 2012 Feb 1;307(5):491-7. http://dx.doi. org/10.1001/jama.2012.39.

3. Londres. National Health Service. Health Survey for England - 2011. 2012 Dec. Disponível em: http://www.ic.nhs.uk/ searchcatalog?productid=10152\&returnid $=1685$

4. Brasil. Ministério da Saúde. VIGITEL Brasil 2006: Vigilância de fatores de risco e proteção para doenças crônicas por inquérito telefônico [acesso em 19 dez 2013]. Disponível em: http://bvsms.saude.

Tabela 1. Custos do SUS com o tratamento ambulatorial e hospitalar com as doenças relacionadas à obesidade (média dos anos de 2008 a 2010).

\begin{tabular}{|c|c|c|c|c|}
\hline \multicolumn{2}{|c|}{ Grupo de doenças } & Custos ambulatoriais ( $\mathrm{R} \$$ ) & Custos hospitalares (R\$) & Custos totais ( $R \$$ ) \\
\hline \multirow{5}{*}{ Cardiovascular } & Cardiovascular (todas) & 148.593 .269 & 1.128 .947 .735 & 1.277 .541 .004 \\
\hline & $\begin{array}{c}\text { Doença arterial coro- } \\
\text { nariana }\end{array}$ & 110.805 .178 & 662.593 .988 & 773.399 .166 \\
\hline & Insuficiência cardíaca & 2.592 .687 & 269.357 .625 & 271.950 .312 \\
\hline & Hipertensão arterial & 23.103 .255 & 37.287 .453 & 60.390 .708 \\
\hline & $\begin{array}{c}\text { Acidente vascular } \\
\text { cerebral }\end{array}$ & 12.092 .150 & 159.708 .669 & 171.800 .819 \\
\hline Neoplasias* & & 407.377 .251 & 102.321 .674 & 509.698 .926 \\
\hline Asma & & 21.188 .655 & 36.847 .320 & 58.035 .974 \\
\hline Diabetes mellitus** & & 1.447 .915 & 37.212 .371 & 40.376 .996 \\
\hline Osteoartrite ${ }^{* * *}$ & & 6.696 .476 & 9.907 .512 & 16.603 .988 \\
\hline
\end{tabular}

* neoplasias relacionadas ao sobrepeso (colorretal, endométrio, ovário, pâncreas, mama, rim, bexiga); **apenas como primeiro diagnóstico; ***apenas joelho e quadril. 
gov.br/bvs/publicacoes/relatorio_vigitel_2006_ marco_2007.pdf

5. Brasil. Ministério da Saúde. VIGITEL Brasil 2010: Vigilância de fatores de risco e proteção para doenças crônicas por inquérito telefônico [acesso em 19 dez 2013]. Disponível em: http://portal.saude. gov.br/portal/arquivos/pdf/vigitel_180411.pdf

6. Schmidt MI, Duncan BB, Silva GA, Menezes AM, Monteiro CA, Barreto SM, et al. Chronic noncommunicable diseases in Brazil: burden and current challenges. Lancet. 2011 Jun;9(11):60135-9. http:// dx.doi.org/10.1016/S0140-6736(11)60135-9

7. ANS.gov.br [homepage na internet]. Rio de Janeiro: Agência Nacional de Saúde Suplementar [acesso em 19 dez 2013]. Disponível em: http://www.ans.gov.br

8. Finkelstein EA, Trogdon JG, Cohen JW, Dietz W. Annual medical spending attributable to obesity: payer-and service-specific estimates. Health Aff (Millwood). 2009 Sep-Oct;28(5):w822-31. http:// dx.doi.org/10.1377/hlthaff.28.5.w822.

9. Hammond RA, Levine R. The economic impact of obesity in the United States. Diabetes Metab Syndr Obes. 2010 Aug;3:285-95. http://dx.doi.org/10.2147/ DMSOTT.S7384

10. Allender S, Rayner M. The burden of overweight and obesity-related ill health in the UK. Obes Rev. 2007 Sep;8(5):467-73.

11. von Lengerke T, Krauth C. Economic costs of adult obesity: A review of recent European studies with a focus on subgroup-specific costs. Maturitas. 2011 Jul;69(3):220-9. http://dx.doi.org/10.1016/j.maturitas.2011.04.005.

12. Konnopka A, Bödemann M, König HH. Health burden and costs of obesity and overweight in Germany. Eur
J Health Econ. 2011 Aug;12(4):345-52. http://dx.doi. org/10.1007/s10198-010-0242-6.

13. Anis AH, Zhang W, Bansback N, Guh DP, Amarsi Z, Birmingham CL. Obesity and overweight in Canada: an updated cost-of-illness study. Obes Rev. 2010 Jan;11(1):31-40. http://dx.doi.org/10.1111/j.1467789X.2009.00579.x.

14. Banco de Preços em Saúde [homepage na internet]. Brasil: Ministério da Saúde [acesso em 9 jan 2014]. Disponível em: http://www.saude.gov.br/bps

15. Oliveira ML. Estimativa dos custos da obesidade para o Sistema Único de Saúde do Brasil [Tese de Doutorado em Nutrição Humana]. Brasília: Universidade de Brasília; 2013.

16. Sichieri R, Nascimento S, Coutinho W. The burden of hospitalization due to overweight and obesity in Brazil. Cad Saúde Pública. 2007 Jul;23(7):1721-7. http:// dx.doi.org/10.1590/S0102-311X2007000700025

17. Bahia L, Coutinho ES, Barufaldi LA, Abreu GA, Malhão TA, de Souza CP, et al. The costs of overweight and obesity-related diseases in the Brazilian public health system: cross-sectional study. BMC Public Health. 2012 Jun 18;12:440. http://dx.doi. org/10.1186/1471-2458-12-440.

18. Guh DP, Zhang W, Bansback N, Amarsi Z, Birmingham $\mathrm{CL}$, Anis AH. The incidence of co-morbidities related to obesity and overweight: a systematic review and meta-analysis. BMC Public Health. 2009 Mar 25;9:88. http://dx.doi.org/10.1186/1471-2458-988.

19. Instituto Brasileiro de Geografia e Estatística. Produto Interno Bruto 2010. Rio de Janeiro: IBGE; 2010. Disponível em: http://www.ibge.gov.br/home/ $\begin{array}{ll}\text { Recebido: } & \text { 19/08/2013. } \\ \text { Revisado: } & \text { 26/11/2013. } \\ \text { Aprovado: } & 04 / 02 / 2014 .\end{array}$ 